\title{
APPLICATION OF MATLAB SYMBOLIC MATHS WITH VARIABLE PRECISION ARITHMETIC (VPA) TO COMPUTE SOME HIGH ORDER GAUSS LEGENDRE QUADRATURE RULES
}

\author{
H.T. Rathod ${ }^{1}$, R.D. Sathish ${ }^{2}$, Md. Shafiqul Islam ${ }^{3}$ and Arun Kumar Gali ${ }^{4}$ \\ ${ }^{1}$ Department of Mathematics, Central college campus, Bangalore University, Bangalore-560 001, India \\ Email: htrathod@yahoo.com \\ ${ }^{2}$ Department of Mathematics, Vijaya Degree college, R.V. Road, Bangalore-560 004, India \\ ${ }^{3}$ Department of Mathematics, University of Dhaka, Dhaka-1000, Bangladesh \\ ${ }^{4}$ Department of Mathematics, Acharya Institute of Technology, Bangalore-560 019, India
}

Received 26.02.08 Accepted 30.08.08

\begin{abstract}
Gauss Legendre Quadrature rules are extremely accurate and they should be considered seriously when many integrals of similar nature are to be evaluated. This paper is concerned with the derivation and computation of numerical integration rules for the three integrals:

(i) $\int_{-1}^{1} f(x) d x$, (ii) $\int_{0}^{1} f(x) / \sqrt{x} d x$; and (iii) $\int_{0}^{1} \sqrt{x} f(x) d x$

which are dependent on the zeros and the squares of the zeros of Legendre Polynomial and is quite well known in the Gaussian Quadrature theory. We have developed the necessary MATLAB programs based on symbolic maths which can compute the sampling points and the weight coefficients and are reported here upto 32 - digits accuracy and we believe that they are reported to this accuracy for the first time. The MATLAB programs appended here are based on symbolic maths. They are very sophisticated and they can compute Quadrature rules of high order, whereas one of the recent MATLAB program appearing in reference [21] can compute Gauss Legendre Quadrature rules upto order twenty, because the zeros of Legendre polynomials cannot be computed to desired accuracy by MATLAB routine roots (.........). Whereas we have used the MATLAB routine solve (........) to find zeros of polynomials which is very efficient. This is worth noting in the present context.
\end{abstract}

\section{Introduction}

It is well known that the n-point Gauss-Legendre Quadrature rule [1]

$$
\int_{-1}^{1} f(x) d x=\sum_{i=1}^{n} w_{i} f\left(x_{i}\right)
$$

has the highest possible precision degree and is analytically exact for polynomials of degree at most $2 n-1$. One can obtain the nodes $x_{i}$ and the weights $w_{i}$ by the method of undermined coefficients, so that by putting the basis functions $f(x)=x^{j}$ in equation (1):

$$
\int_{-1}^{1} x^{j} d x=\sum_{i=1}^{n} w_{i} x_{i}^{j} \quad j=1,2, \cdots \cdots, 2 n-1
$$


it leads to a nonlinear system of equations with $2 \mathrm{n}$ unknowns. The following theorem shows the complete formula of Gauss Legendre Quadrature rule [2].

\section{Theorem :}

Let $f(x) \in C^{2 n}[-1,1]$, then

$$
\int_{-1}^{1} f(x) d x=\sum_{i=1}^{n} w_{i} f\left(x_{i}\right)+E(f)
$$

where $x_{i}$ denotes the $i$-th zero of the Legendre polynomial $P_{n}(x)$ and the weight coefficients $w_{i}$ and the remainder term $E(f)$ are given by:

$$
\begin{aligned}
& w_{i}=\frac{2}{\left(1-x_{i}^{2}\right)\left[P_{n}^{\prime}\left(x_{i}\right)\right]^{2}}=\frac{2\left(1-x_{i}^{2}\right)}{n^{2}\left[P_{n-1}\left(x_{i}\right)\right]^{2}}=\frac{-2}{(n-1) P_{n+1}\left(x_{i}\right) P_{n}^{\prime}\left(x_{i}\right)} \\
& E(f)=\frac{2^{2 n+1}}{(2 n+1)} \frac{(n !)^{4}}{[(2 n) !]^{3}} f^{(2 n)}(\eta),-1<\eta<1
\end{aligned}
$$

In this paper we are concerned with a general n-point Gauss-Legendre Quadrature rule, since a system of nonlinear equations like (2) can be very difficult to solve as the dimension increases. But by virtue of the above theorem, the n-nodes can be obtained as zeros of the $\mathrm{n}^{\text {th }}$ degree Legendre polynomial, which can be generated by any of the following three equations:

$$
P_{i}(x)=\sum_{k=0}^{M}(1)^{k} \frac{(2 n-2 k) !}{2^{n}(k !)(n-k) !(n-2 k) !} x^{n-2 k}
$$

where $M=n / 2$ if $n$ is even, and $M=(n-1) / 2$ if $n$ is odd

$$
\begin{aligned}
& P_{n}(x)=\left[(2 n-1) x P_{n-1}(x)-(n-1) P_{n-2}(x)\right] / n, \quad n=2,3, \cdots \cdots \\
& P_{0}(x)=1, P_{1}(x)=1, \\
& P_{n}(x)=\frac{1}{2^{n}(n !)} \quad \frac{d^{n}}{d x^{n}}\left(x^{2}-1\right)^{n}
\end{aligned}
$$

Given the $n$-nodes $x_{i}(i=1,2, \ldots \ldots \ldots, n)$, we can get the corresponding weight coefficients $w_{i}$ either by equation (4) or by solving the system of linear equations as in [3].

$$
\left[\begin{array}{lllll}
1 & 1 & 1 & \cdots \cdots & 1 \\
x_{1} & x_{2} & x_{3} & \cdots \cdots & x_{n} \\
x_{1}^{2} & x_{2}^{2} & x_{3}^{2} & \cdots \cdots & x_{n}^{2} \\
\cdots & \cdots & \cdots & \cdots \cdots & \cdots \\
x_{1}^{n-1} & x_{2}^{n-1} & x_{3}^{n-1} & \cdots \cdots & x_{n}^{n-1}
\end{array}\right]\left[\begin{array}{l}
w_{1} \\
w_{2} \\
w_{3} \\
\vdots \\
w_{n}
\end{array}\right]=\left[\begin{array}{c}
2 \\
0 \\
2 / 3 \\
\vdots \\
{\left[1-(-1)^{n}\right] / n}
\end{array}\right]
$$

which follows from equation (2) for $j=0,1,2, \ldots \ldots \ldots, n-1$. 
However, there is yet another method based on the evaluation of integrals, which again follows from the theory on numerical integration, the following theorem may be recalled for this purpose [8-13].

\section{Theorem :}

Let $f \in C^{n+1}[a, b]$ and distinct nodes $x_{i}, i=1,2, \cdots \cdots, n$, the Lagrange interpolation holds and we have,

$$
f(x)=\sum_{i=1}^{n} l_{i}\left(x_{i}\right) f\left(x_{i}\right)+\frac{f^{(n)}(\eta(x))}{n !} \psi_{n}(x), \quad \eta(x) \in C[a, b]
$$

in which

$$
l_{i}(x)=\prod_{\substack{k=1 \\ k \neq i}}^{n} \frac{\left(x-x_{k}\right)}{\left(x_{i}-x_{k}\right)}
$$

is the Lagrange interpolant polynomial dependent on $x_{1}, x_{2}, \cdots, x_{n}$ and

$$
\psi_{n}(x)=\prod_{i=1}^{n}\left(x-x_{i}\right)
$$

Then it follows that

$$
\int_{a}^{b} f(x) w(x) d x=\sum_{k=1}^{n} w_{k} f\left(x_{k}\right)+E_{n}(f)
$$

where,

$$
w_{k}=\int_{a}^{b} l_{k}(x) w(x) d x \quad, E_{n}(f)=\int_{a}^{b} \frac{1}{n !} f^{(n+1)}(\eta(x)) \psi_{n}(x) w(x) d x
$$

From the above theorem, with $a=-1, b=1, w(x)=1, x_{i}(i=1,2, \cdots \cdots, n)$ as distinct zeros of $n$-th order Legendre polynomial. The integration formula (9) and (10) can be shown to be equivalent to the formula in eqns (3-5). It is clear that in this case for $w(x)=1$.

$$
w_{k}=\int_{-1}^{1} l_{k}(x) d x
$$

This paper is concerned with the determination of zeros and the squares of zeros of the Legendre Polynomials which will be necessary to find numerical integration formulas for the following integrals in addition to the one stated in equation (1):

$$
\int_{0}^{1} \frac{f(x)}{\sqrt{x}} d x
$$




$$
\int_{0}^{1} \sqrt{x} f(x) d x
$$

We shall now further elaborate on the procedure for the above two integrals of eqns (12) $\&(13)$.

The Integral $\int_{0}^{1} \frac{f(x)}{\sqrt{x}} d x$

We have $\int_{0}^{1} \frac{f(x)}{\sqrt{x}} d x=\int_{0}^{1} \frac{f\left(y^{2}\right)}{y} 2 y d y$

$$
=2 \int_{0}^{1} f\left(y^{2}\right) d y=\int_{0}^{1} f\left(y^{2}\right) d y+\int_{0}^{1} f\left(y^{2}\right) d y
$$

Since, $\int_{0}^{1} f\left(y^{2}\right) d y=\int_{0}^{1} f\left(\left(-Y^{2}\right)\right)(-d Y)=-\int_{0}^{1} f\left(Y^{2}\right) d Y=\int_{-1}^{0} f\left(y^{2}\right) d y$

Therefore $\int_{0}^{1} \frac{f(x)}{\sqrt{x}} d x=\int_{-1}^{1} f\left(y^{2}\right) d y=\int_{-1}^{1} f\left(x^{2}\right) d x$

$$
\begin{aligned}
& =\sum_{i=1}^{2 m} w_{i} f\left(y_{i}^{2}\right)=\sum_{i=1}^{m} w_{i} f\left(y_{i}^{2}\right)+\sum_{i=1}^{m} w_{i} f\left(\left(-y_{i}\right)^{2}\right), n=2 m \\
& =2 \sum_{i=1}^{m} w_{i} f\left(y_{i}^{2}\right)=2 \sum_{i=1}^{m} w_{i} f\left(x_{i}^{2}\right)=\sum_{i=1}^{m}\left(2 w_{i} f\left(x_{i}^{2}\right)\right.
\end{aligned}
$$

where $x_{i}^{2}$ are the squares of the zeros of $P_{2 m}(x)$, here the integrand is singular at one of the points. In this case, the orthogonal polynomial of degree $m$ is $P_{2 m}(\sqrt{x})$ where, $P_{2 m}(x)$ is the Legendre Polynomial of degree $2 m$ corresponding to each positive zero $x_{i}$ of $P_{2 m}(x)$, there is an abscissa given by $x_{i}^{2}$ and the corresponding weight is $2 w_{i}$, where $w_{i}$ is the weight corresponding to $x_{i}$.

The truncation error in this case can be expressed as [8]

$$
E_{m}=\frac{2^{4 m+1}[(2 m) !]^{2}}{(4 m+1)[(4 m) !]^{2}} f^{(2 m)}(\eta), \quad-1<\eta<1
$$

The Integral $\int_{0}^{1} \sqrt{x} f(x) d x$ : 
We have

$$
\begin{aligned}
\int_{0}^{1} \sqrt{x} f(x) d x & =\int_{0}^{1} \sqrt{y^{2}} f\left(y^{2}\right) 2 y d y=2 \int_{0}^{1} y^{2} f\left(y^{2}\right) d y=\int_{-1}^{1} y^{2} f\left(y^{2}\right) d y \\
& =\sum_{i=0}^{2 m} w_{i} f\left(y_{i}^{2}\right)=\sum_{i=0}^{2 m} w_{i} x_{i}^{2} f\left(x_{i}^{2}\right)=\sum_{i=1}^{2 m} w_{i} x_{i}^{2} f\left(x_{i}^{2}\right)=\sum_{i=1}^{m} 2 w_{i} x_{i}^{2} f\left(x_{i}^{2}\right)
\end{aligned}
$$

where $x_{0}=0, w_{0} \neq 0$ and $x_{1}, x_{2}, \cdots \cdots, x_{2 m}$ are the zeros of $P_{2 m+1}(x)$ and $x_{i}^{2},(i=1,2, \cdots \cdots, m)$ are the zeros of $\frac{P_{2 m+1}(\sqrt{x})}{\sqrt{x}}$ and $w_{i}$ are the weights corresponding to $x_{i}$. The truncation error is given by [8].

$$
E_{m}=\frac{2^{4 m+3}[(2 m+1) !]^{4}}{(4 m+3)[(4 m+2) !]^{2}(2 m) !} f^{(2 m)}(\eta),-1<\eta<1
$$

But the authors have not come across any reference which tabulates the squares of the zeros of Legendre Polynomials.

We may also note that the computation of Gaussian Quadrature rules in general involves two distinct phases;

(i) The generation of the orthogonal polynomials (ex : Legendre, Jacobi, etc.,) say $P_{0}, P_{1}, \cdots \cdots, P_{n}$.

(ii) The determination of zeros of $P_{n}(x)$, and the computation of the associated weights.

Phase (i) may appear trivial, but it is not so for large values of $n$.

Phase (ii) is really difficult for values of $n$ larger than, say 10 .

We may note the following facts regarding zeros of Legendre polynomials.

(i) It is well known that all zeros of Legendre polynomials are real and distinct and lie in the interval $(-1,1)$. Hence the existence of a high order quadrature formula is assured for all values of $n$.

(ii) It should be noted that the zeros of Legendre polynomials are distributed symmetrically about $x=0$ and if the abscissas (nodes) are arranged in a monotonic sequence, then $\quad x_{i}=-x_{n+1-i}, w_{i}=w_{n+1-i}(i=1,2, \cdots, n)$

In this paper, we have generated the Legendre polynomial by using symbolic Maths of MATLAB software [12-21]. We also note here with great surprise that MATLAB builtin function roots (.....) fails to find roots to a reasonable accuracy even for $10<n<15$, and it fails to satisfy the properties listed above for zeros of Legendre polynomials, we are also surprised to find that roots (.....) function gives some of the zeros as complex conjugates. However, we succeeded in determining zeros up to 32 digits accuracy by using the powerful built-in function solve (.....) available in the MATLAB programming. 
On comparing with tabulated zeros [4], the accuracy of the results by solve (.....) function are confirmed. But we may note that the tabulated zeros are shown in [4-7] to at most 20 digits accuracy.

However, the authors have not come across any reference which tabulates the sampling points and weight coefficients of other two integrals $\int_{0}^{1} f(x) / \sqrt{x} d x$ and $\int_{0}^{1} \sqrt{x} f(x) d x$.

For even values of $n$, we have $n / 2$ pairs of abscissas with opposite signs, while for odd values of $n$, there are $(n-1) / 2$ pairs and hence only half the values need to be tabulated in tables of weights and abscissas of Gauss Legendre quadrature formulas, such tables are available in [4-7]. They are printed with 15-digits of accuracy for orders 2, 3, 4, 5, 6, 7, 8, $9,10,12$. Then there after for orders $16,20,24,32,40,48,64,80$ and 96 with 20-digit accuracy. We note that there may be a necessity of other remaining orders and also there might be a need for accuracy with more number of digits. This paper aims at providing the high order Gauss Legendre Quadrature rules for three types of integrals with variable precision arithmetic up to 32-digits accuracy. Because of memory constraints, we could obtain good results up to order 48, beyond this order the results appear to have less accuracy. But the zeros and squares of zeros are computable with great accuracy even beyond order 96 using the built-in function solve (.....) by the computer programs, which are available upon the request to the corresponding author.

\section{Method of undetermined coefficients}

We shall now determine the weight coefficients for the above stated integrals. First of all the weights coefficients could be easily obtained by equation (4). But we notice that for large $\mathrm{n}$, we fail to determine the coefficients due to numerical instability. Next we shall consider the method of equation (5). We find that solution of linear equations also fails due to singular matrices. We can surmount this problem by considering the solution as two sets of linear systems as explained below.

(i) Weight coefficients for odd order Gauss Legendre Quadrature rules:

We may note that the abscissas (nodes) and weight coefficients satisfy the relation.

$$
x_{i}=-x_{(n-1) / 2+i}, w_{i}=w_{(n-1) / 2+i}(i=1,2, \cdots,(n-1) / 2,(n-1) / 2+1
$$

On using the above equations for determining $w_{i}$ 's:

$$
\sum_{i=1}^{(n-1) / 2} w_{i} x_{i}^{2 j}=\frac{1}{2 j+1} \quad j=1,2, \cdots \cdots,(n-1) / 2
$$

and

$$
w_{(n-1) / 2+1}=2\left[1-\sum_{i=1}^{(n-1) / 2} w_{i}\right]
$$


The linear system (19a) needs squares of zeros of the Legendre polynomials of order $n$ as input. The weight coefficients corresponding to the zero $x_{(n-1) / 2+1}=0$ is $w_{(n-1) / 2+1}$ and this can be obtained from equation (19b). We see that this requires nearly half the number of equations listed in equation (6). This is implemented in function gausswtsodd.m. The outcome of this function is very encouraging.

\section{(ii) Weight coefficients for even order Gauss Legendre Quadrature rules:}

In this case we note that the abscissas (nodes), $x_{i}$ and the corresponding weight coefficients, $w_{i}$ satisfy the relations :

$$
X_{i}=-X_{n / 2+i}, W_{i}=w_{n / 2+i}(i=1,2, \cdots, n / 2
$$

On using the above relations, we obtain the following linear system of equations.

$$
\sum_{i=1}^{n / 2} W_{i} X_{i}^{2(j-1)}=\frac{1}{2 j-1} \quad j=1,2, \cdots \cdots, n / 2
$$

Again, it may be noted that the input to the above linear system again requires the squares of the zeros of corresponding Legendre polynomial of order $\mathrm{n}$. This is implemented in function gausswtseven.m. Again, we wish to state that the outcome of this function is also very encouraging.

\section{Integration Method to determine weight coefficients}

The weight coefficients can also be directly found by the formula of equation (11)

$$
W_{k}=\int_{0}^{1} \frac{\left(x-x_{1}\right)\left(x-x_{2}\right) \cdots \cdots\left(x-x_{k-1}\right)\left(x-x_{k+1}\right) \cdots\left(x-x_{n}\right)}{\left(x_{k}-x_{1}\right)\left(x_{k}-x_{2}\right) \cdots \cdots\left(x_{k}-x_{k-1}\right)\left(x_{k}-x_{k+1}\right) \cdots\left(x_{k}-x_{n}\right)} d x
$$

Using the convention stated in the previous section regarding abscissas (zero) and weight coefficients, we obtain the following.

\section{(i) Odd order - Gauss Legendre Quadrature rules :}

From equations (18), (19a - b) and (22), we may write

$$
\begin{aligned}
& W_{k}=\int_{0}^{1} \frac{\sqrt{T}\left(T-X_{1}\right)\left(T-X_{2}\right) \cdots \cdots\left(T-X_{k-1}\right)\left(T-X_{k+1}\right) \cdots\left(T-X_{n}\right)}{2 X_{k}\left(X_{k}-X_{1}\right)\left(X_{k}-X_{2}\right) \cdots \cdots\left(X_{k}-X_{k-1}\right)\left(X_{k}-X_{k+1}\right) \cdots\left(X_{k}-X_{n}\right)} d T \\
& \text { with }(k=1,2, \ldots ., N ; N=(n-1) / 2) \text { and } X_{i}=x_{i}^{2} \text { and } w_{0}=2\left[1-\sum_{i=1}^{(n-1) / 2} w_{i}\right]
\end{aligned}
$$

\section{(ii) Even order - Gauss Legendre Quadrature rules:}

From equations (20), (21) and (22), we find that: 


$$
\begin{aligned}
& W_{k}=\int_{0}^{1} \frac{T^{-1 / 2}\left(T-X_{1}\right)\left(T-X_{2}\right) \cdots\left(T-X_{k-1}\right)\left(T-X_{k+1}\right) \cdots\left(T-X_{n}\right)}{2 X_{k}\left(T_{k}-X_{1}\right)\left(T_{k}-X_{2}\right) \cdots \cdots\left(T_{k}-X_{k-1}\right)\left(T_{k}-X_{k+1}\right) \cdots\left(T_{k}-X_{n}\right)} d T \\
& \text { with }(k=1,2, \ldots \ldots, N ; N=n / 2) \text { and } X_{i}=x_{i}^{2}
\end{aligned}
$$

\section{Numerical results}

Some sample outputs from the appended MATLAB program in M-file named legendrepoly.m, for $\mathrm{n}=20,21,40,41,48,49$, which are available upon request to the corresponding authour, since it has been omitted to reduce the length of the paper.

\section{Conclusion}

In this paper, we have presented the numerical integration rules for the following integrals; $\int_{-1}^{1} f(x) d x, \int_{0}^{1} f(x) / \sqrt{x} d x$ and $\int_{0}^{1} \sqrt{x} f(x) d x$. The rules are known as $n$-point Gauss Legendre Quadrature rules for the integrals $\int_{0}^{1} f(x) d x \cong \sum_{i=1}^{n} w_{i} f\left(x_{i}\right)$ and are analytically exact for polynomials of degree at most $2 n-1$, where $x_{i}$ are the zeros of $P_{n}(x)$ and $w_{i}$ the corresponding weight coefficients. Other two integrals $\int_{0}^{1} f(x) / \sqrt{x} d x$ and $\int_{0}^{1} \sqrt{x} f(x) d x$ are shown to be equivalent to the integrals $\int_{-1}^{1} x^{2} f\left(x^{2}\right) d x$ and $\int_{-1}^{1} f\left(x^{2}\right) d x$ respectively and they also have a precision of degree $2 n-1$ for the n-point formulas, but the formulas

$$
\begin{array}{ll}
\int_{0}^{1} f(x) / \sqrt{x} d x \cong \sum_{i=1}^{m}\left(2 w_{i}\right) f\left(x_{i}{ }^{2}\right), & m=n / 2 \\
\int_{0}^{1} \sqrt{x} f(x) d x \cong \sum_{i=1}^{m}\left(2 w_{i}{x_{i}}^{2}\right) f\left(x_{i}{ }^{2}\right), & m=(n-1) / 2
\end{array}
$$

have their nodes $x_{i}$ 's as the zeros of $P_{2 n}(\sqrt{x})$ and $P_{2 n+1}(\sqrt{x}) / \sqrt{x}$ respectively i.e., they are the squares of the zeros of $P_{2 n}(x)$ and $P_{2 n+1}(x)$ respectively.

Extensive tables of numerical integration rules for $\int_{-1}^{1} f(x) d x$ are available in the literature, but they are tabulated to either upto 15 or 20 digit accuracy and they are only tabulated for selected orders;

$$
n=2(1) 10,12,15,16,20,24,30,32,40,48,64,80,96 .
$$

But Gauss Legendre Quadrature rules of odd order are very scarcely tabulated. However, it is very surprising to know that for integrals $\int_{0}^{1} f(x) / \sqrt{x} d x$ and $\int_{0}^{1} \sqrt{x} f(x) d x$ and the table of formulas are not available, at least to the knowledge of the present authors.

In this paper, we have applied MATLAB symbolic maths with variable precision arithmetic (vpa) to perform all the programming tasks either analytical or numerical, which helped us to compute the integration formulas considered here and the necessary 
MATLAB computer program which can compute abscissas and weight coefficients of Gauss Legendre Quadrature rule for any order $n$ is appended here for ready reference. We have also found that the appended program can compute the numerical integration formulas of orders ; $1 \leq n \leq 48$ which agree with standard tables presented in [4-7]. We hope that the MATLAB program presented here will be a useful addition to the existing software on numerical integration. We have appended herewith some sample output of Gauss Quadrature Rules for $n=40,41,48$ and 49 for the integrals $\int_{-1}^{1} f(x) d x$ and the corresponding integrals $\int_{0}^{1} \sqrt{x} f(x) d x$ and $\int_{0}^{1} f(x) / \sqrt{x} d x$.

\section{REFERENCES}

1. A Stroud and D. Secrest, Gaussian Quadrature Formulas, Practice Hall, 1966, Englewood cliffs, New Jersey, (1966).

2. P. Davis and P. Rabinowiz, Methods of numerical integration, $2^{\text {nd }}$ edition, Academic Press, New York, (1984).

3. Krylov, V.I, Approximate Calculation of integrals (Trans. A. H. Stroud), McMillan New York, (1962).

4. M. Abramowitz and I. Stegun, Eds. Handbook of Mathematical Functions, Washington D.C, National Bureau of Standards, 1964, Reprinted by Dover publication, New York, 916-919, (1968).

5. Davis P. Rabinouitz. P, Abscissas and weights for Gaussian Quadrature of high order; J. Res. Nat. Bur. Stand, 56, 35-37, (1956).

6. Davis. P and Rabinowitz. P, Additional abscissas and weights for Gaussian Quadratures of high order: values for $\mathrm{n}=64,80$ and 1996. J. res. Nat. Bur. stand. 60, 613-614, (1958).

7. Lowan. A.N., Davids. N, Levenson.A, Tables of the zeros of the Legendre polynomials of order 1-16 and the weight coefficients for Gauss Mechanical Quadrature formula, Buss Amar. Math. Soc. 48, 739743, (1942).

8. K. Atkinson, An Introduction to numerical analysis, $2^{\text {nd }}$ Edition, John Willey, New York, (1993).

9. Carnahan. B, Luther. H.A and Wilkes, J.O., Applied Numerical Methods, John Wiley, New York, (1969).

10. S. Zhang and J. Jinn, computation of special functions, John Wiley, (1996).

11. Burden, R.L and Fairs, J. L, Numerical Analysis, $7^{\text {th }}$ Edition, Books/cole Thomson, Pacific Grove, C.A, (2001).

12. Gerald, C.F and Wheatley, P.O, Applied Numerical Analysis, $3^{\text {rd }}$ Edition, Addison Wesley, Reading, MA, (1984).

13. Mathews, J.H and Fink. K, Numerical Methods using MATLAB, $3^{\text {rd }}$ Edition, Prentice Hall, Wpper Saddle River, New Jersey, NJ, (1999).

14. Haint B.R, Ronald. L.L and Jonathan. M.R, A guide to MATLAB for beginners and experienced users, Cambridge University Press, (2001).

15. Hanselman, D and Bruce. L, Mastering MATLAB ${ }^{\circledR}$ Pearson Education, (2005).

16. Chapman. S.J, MATLAB ${ }^{\circledR}$ programming for engineers, $3^{\text {rd }}$ Edition, Thomson Learning, (2004).

17. Gilat, A., MATLAB ${ }^{\circledR}$ An Introduction with applications, John Willey and Sons Inc., (2004).

18. Otto. S.R and Denier. J.P, An Introduction to programming and numerical methods in MATLAB, Springer International Edition, Springer-Verlag London Limited, (2005).

19. Kirani Singh. Y and Chandhuri. B. B, MATLAB programming, Prentice Hall India Private Limited, (2007).

20. Stanley. W. D. Technical Analysis and Applications with MATLAB, Thomson Learning, (2007).

21. Yang W. Y, Cao. W, T.S Chung and Morris. J., Applied Numerical Method using MATLAB, John Wiley and Sons, (2006). 